7th International Workshop on Astronomy and

Relativistic Astrophysics (IWARA 2016)

International Journal of Modern Physics: Conference Series

Vol. 45 (2017) 1760067 (7 pages)

(C) The Author(s)

DOI: $10.1142 / S 2010194517600679$

\title{
Low Energy KN Interaction in the Fock-Tani Formalism
}

\author{
Bruna Cesira Folador and Dimiter Hadjimichef \\ Instituto de Física \\ Universidade Federal do Rio Grande do Sul \\ Av. Bento Gonçalves, 9500 \\ Porto Alegre, Rio Grande do Sul, 91501-970, Brazil \\ bru.folador@gmail.com,dimihadj@gmail.com
}

Published 15 August 2017

\begin{abstract}
The Fock-Tani formalism is a first principle method to obtain effective interactions from microscopic Hamiltonian. Originally derived for meson-meson or baryon-baryon scattering, we present the corresponding equations for meson-baryon scattering. In particular we shall obtain the low energy cross sections for the $K^{-}+p \rightarrow \Lambda+\eta$ channel.

Keywords: Fock-Tani Formalism, Non-Relativistic Quark Model, Kaon-Baryon Interaction.

PACS numbers: 12.39.Jh; 12.39.Pn; 13.75.Jz
\end{abstract}

\section{Fock-Tani Formalism}

There are many examples of systems in which the internal degrees of freedom of composite particles can not be neglected. For these systems the second quantization formalism is a very difficult mathematical tool to be used. Alternatively, one can map the physical Hilbert space into an ideal Hilbert space, where the boundstates are represented by "ideal particles" in which the elementary creation and annihilation operators, obey canonical commutation rules. The information about its internal structure is transferred to an effective interaction Hamiltonian ${ }^{1-3}$. This formalism is called the Fock-Tani formalism.

The starting point of the Fock-Tani formalism is the definition of single composite bound states. We write a single-meson state in terms of a meson creation operator as $|\alpha\rangle=M_{\alpha}^{\dagger}|0\rangle$, where $|0\rangle$ is the vacuum state. The meson creation operator is written in terms of constituent quark and antiquark creation operators $q^{\dagger}$

This is an Open Access article published by World Scientific Publishing Company. It is distributed under the terms of the Creative Commons Attribution 4.0 (CC-BY) License. Further distribution of this work is permitted, provided the original work is properly cited. 
and $\bar{q}^{\dagger}$

$$
M_{\alpha}^{\dagger}=\Phi_{\alpha}^{\mu \nu} q_{\mu}^{\dagger} \bar{q}_{\nu}^{\dagger}
$$

here $\Phi_{\alpha}^{\mu \nu}$ is the meson wave function and $q_{\mu}|0\rangle=\bar{q}_{\nu}|0\rangle=0$. The index $\alpha$ identifies the meson quantum numbers of space, spin and isospin. The indices $\mu$ and $\nu$ denote the spatial, spin, flavor, and color quantum numbers of the constituent quarks. A sum over repeated indices is implied.

In a similar way, the baryon state can be defined as $|\alpha\rangle=B_{\alpha}^{\dagger}|0\rangle$, where the baryon creation operator is

$$
B_{\alpha}^{\dagger}=\frac{1}{\sqrt{6}} \Psi_{\alpha}^{\mu_{1} \mu_{2} \mu_{3}} q_{\mu_{1}}^{\dagger} q_{\mu_{2}}^{\dagger} q_{\mu_{3}}^{\dagger}
$$

$\Psi_{\alpha}^{\mu_{1} \mu_{2} \mu_{3}}$ is the baryon wave function, where the index $\alpha$ identifies the quantum numbers of the baryon, and $\mu$ those of the quarks.

The Fock-space amplitude is taken to be orthonormalized,

$$
\Phi_{\alpha}^{* \mu \nu} \Phi_{\beta}^{\mu \nu}=\delta_{\alpha \beta} \quad ; \quad \Psi_{\alpha}^{* \mu_{1} \mu_{2} \mu_{3}} \Psi_{\beta}^{\mu_{1} \mu_{2} \mu_{3}}=\delta_{\alpha \beta}
$$

The quark and antiquark operators satisfy canonical anti-commutation relations,

$$
\begin{gathered}
\left\{q_{\mu}, q_{\nu}^{\dagger}\right\}=\left\{\bar{q}_{\mu}, \bar{q}_{\nu}^{\dagger}\right\}=\delta_{\mu \nu}, \\
\left\{q_{\mu}, q_{\nu}\right\}=\left\{\bar{q}_{\mu}, \bar{q}_{\nu}\right\}=\left\{q_{\mu}, \bar{q}_{\nu}\right\}=\left\{q_{\mu}, \bar{q}_{\nu}^{\dagger}\right\}=0 .
\end{gathered}
$$

Using these quark anti-commutation relations, and the normalization condition of Eq. (3), it is easily shown that the operators satisfy the following non-canonical (anti-) commutation relations

$$
\begin{aligned}
& {\left[M_{\alpha}, M_{\beta}^{\dagger}\right]=\delta_{\alpha \beta}-\Delta_{\alpha \beta}^{M},} \\
& \left\{B_{\alpha}, B_{\beta}^{\dagger}\right\}=\delta_{\alpha \beta}-\Delta_{\alpha \beta}^{B},
\end{aligned}
$$

where

$$
\begin{gathered}
\Delta_{\alpha \beta}^{M}=\Phi_{\alpha}^{* \mu \nu} \Phi_{\beta}^{\mu \sigma} \bar{q}_{\sigma}^{\dagger} \bar{q}_{\nu}+\Phi_{\alpha}^{* \mu \nu} \Phi_{\beta}^{\rho \nu} q_{\rho}^{\dagger} q_{\mu} \\
\Delta_{\alpha \beta}^{B}=3 \Psi_{\alpha}^{* \mu_{1} \mu_{2} \mu_{3}} \Psi_{\beta}^{\mu_{1} \mu_{2} \nu_{3}} q_{\nu_{3}}^{\dagger} q_{\mu_{3}}-\frac{3}{2} \Psi_{\alpha}^{* \mu_{1} \mu_{2} \mu_{3}} \Psi_{\beta}^{\mu_{1} \nu_{2} \nu_{3}} q_{\nu_{3}}^{\dagger} q_{\nu_{2}}^{\dagger} q_{\mu_{2}} q_{\mu_{3}} .
\end{gathered}
$$

The presence of the operator $\Delta_{\alpha \beta}$ in Eq. (1) is due to the composite nature of the hadrons. This term enormously complicates the mathematical description of processes that involve the hadron and quark degrees of freedom. The operators $M_{\alpha}$, $M_{\alpha}^{\dagger}$ and $B_{\alpha}, B_{\alpha}^{\dagger}$ are not convenient dynamical variables to be used. 
A transformation is defined such that a single-hadron state $|\alpha\rangle$ is redescribed by an ("ideal") elementary-hadron state by

$$
U_{M}^{-1} M_{\alpha}^{\dagger}|0\rangle=m_{\alpha}^{\dagger}|0\rangle \quad ; \quad U_{B}^{-1} B_{\alpha}^{\dagger}|0\rangle=b_{\alpha}^{\dagger}|0\rangle,
$$

where $m_{\alpha}^{\dagger}$ and $b_{\alpha}^{\dagger}$ are the ideal meson/baryon creation operators. The ideal particle operators satisfy, by definition, canonical (anti-) commutation relations

$$
\begin{gathered}
{\left[m_{\alpha}, m_{\beta}^{\dagger}\right]=\delta_{\alpha \beta} \quad ; \quad\left[m_{\alpha}, m_{\beta}\right]=0,} \\
\left\{b_{\alpha}, b_{\beta}^{\dagger}\right\}=\delta_{\alpha \beta} \quad ; \quad\left\{b_{\alpha}, b_{\beta}\right\}=0 .
\end{gathered}
$$

In addition, in the new representation the quark and antiquark operators $q^{\dagger}, q, \bar{q}^{\dagger}$ and $\bar{q}$ are kinematically independent of $m_{\alpha}^{\dagger}, m_{\alpha}, b_{\alpha}^{\dagger}$ and $b_{\alpha}$

$$
\begin{gathered}
{\left[q_{\mu}, m_{\alpha}\right]=\left[q_{\mu}, m_{\alpha}^{\dagger}\right]=\left[\bar{q}_{\mu}, m_{\alpha}\right]=\left[\bar{q}_{\mu}, m_{\alpha}^{\dagger}\right]=0,} \\
\left\{q_{\mu}, b_{\alpha}\right\}=\left\{q_{\mu}, b_{\alpha}^{\dagger}\right\}=0 .
\end{gathered}
$$

The unitary operators in (7) are defined by

$$
U_{M}(t)=\exp \left[t F_{M}\right] \quad ; \quad U_{B}(t)=\exp \left[t F_{B}\right]
$$

where $F_{M}$ is the generator of the transformation and $t$ a parameter which is set to $-\pi / 2$ to implement the mapping. The generators $F_{M}$ and $F_{B}$ of the transformations are

$$
F_{M}=m_{\alpha}^{\dagger} \tilde{M}_{\alpha}-\tilde{M}_{\alpha}^{\dagger} m_{\alpha} \quad ; \quad F_{B}=b_{\alpha}^{\dagger} \tilde{B}_{\alpha}-\tilde{B}_{\alpha}^{\dagger} b_{\alpha},
$$

where

$$
\tilde{M}_{\alpha}=\sum_{i=0}^{n} \tilde{M}_{\alpha}^{(i)}, \quad ; \quad \tilde{B}_{\alpha}=\sum_{i=0}^{n} \tilde{B}_{\alpha}^{(i)} .
$$

We need a transformation such that a single-hadron state $|\alpha\rangle$ is redescribed by an ("ideal") elementary-hadron state. In the present calculation we obtain a mixed meson-baryon transformation defined by

$$
O_{\alpha}\left(t, t^{\prime}\right)=U_{B}^{-1} U_{M}^{-1} O_{\alpha} U_{M} U_{B}
$$

Once a microscopic interaction Hamiltonian $H$ is defined, at the quark level, a new transformed Hamiltonian can be obtained. The transformed Fock-Tani Hamiltonian is a result of the application of the unitary transformation on the microscopic Hamiltonian

$$
H_{\mathrm{FT}}=U_{B}^{-1} U_{M}^{-1} H U_{M} U_{B}
$$

The transformed Hamiltonian $H_{\mathrm{FT}}$ describes all possible processes involving mesons, baryons and quarks. After the applying the Fock-Tani transformation we obtain the 


\section{B. C. Folador \& D. Hadjimichef}

following general meson-baryon potential

$$
V_{\mathrm{mb}}(\alpha \beta ; \delta \gamma)=\sum_{i=1}^{4} V_{i}(\alpha \beta ; \delta \gamma) m_{\alpha}^{\dagger} b_{\beta}^{\dagger} m_{\gamma} b_{\delta},
$$

where

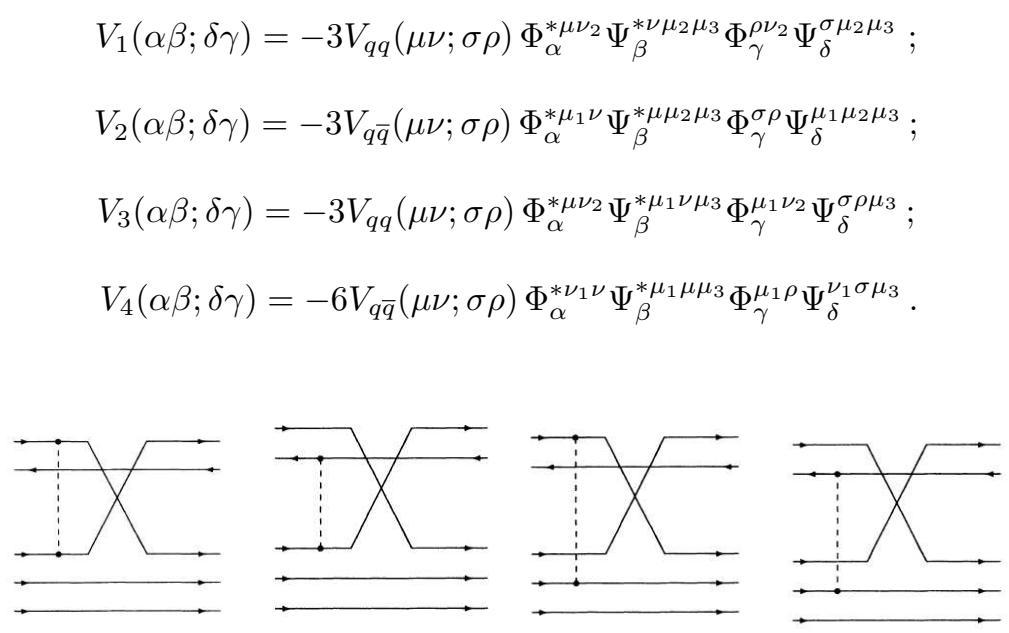

Fig. 1. Scattering diagrams $V_{1}, V_{2}, V_{3}$ and $V_{4}$ respectively.

\section{Differential Cross-Section}

The interaction potential between quarks is

$$
\begin{aligned}
V_{q q}= & \sum_{i j}\left[\frac{\alpha_{s}}{r_{i j}}-\frac{3}{4} b r_{i j}-\frac{8 \alpha_{s}}{3 \sqrt{\pi} m_{i} m_{j}} \sigma_{1}^{3} e^{-\sigma_{1}^{2} r_{i j}^{2}}\left(\vec{S}_{i} \cdot \vec{S}_{j}\right)\right. \\
& \left.-\frac{\pi \alpha_{s}}{2} \sigma_{2}^{3} e^{-\sigma_{2}^{2} r_{i j}^{2}}\left(\frac{1}{m_{i}^{2}}-\frac{1}{m_{j}^{2}}\right)\right]\left(\vec{T}_{i} \cdot \vec{T}_{j}\right) .
\end{aligned}
$$

In the Born approximation, the matrix element $\left\langle\alpha \beta\left|V_{\mathrm{mb}}\right| \gamma \delta\right\rangle$ between ideal states results in the following S-matrix

$$
S_{f i}=\delta_{f i}-2 \pi i \delta\left(E_{f}-E_{i}\right) V_{\mathrm{mb}}(\alpha \beta ; \delta \gamma) .
$$

Due to translational invariance, one can write $V_{\mathrm{mb}}$ in (17) as

$$
V_{\mathrm{mb}}(\alpha \beta ; \delta \gamma)=\delta\left(P_{f}-P_{i}\right) h_{f i},
$$

and the scattering amplitude $h_{f i}$ is defined by

$$
h_{f i}=\sum_{k=1}^{4} V_{k} .
$$


The diagrams $V_{k}$ can be seen in figure 1 and can be written as

$$
V_{k}=\omega_{k} I_{k}^{e},
$$

where $I_{k}^{e}$ are the spatial integrals and $\omega_{k}$ the spin-flavor-color part. These amplitudes can be related to the cross section in the center of mass system ${ }^{4}$

$$
\frac{d \sigma}{d \Omega}=\frac{1}{64 \pi^{2} s} \frac{\left|\overrightarrow{p_{f}}\right|}{\left|\overrightarrow{P_{k}}\right|}|\mathcal{M}|^{2},
$$

where $\vec{P}_{k}$ and $\vec{p}_{f}$ are the initial and final state momenta. The invariant amplitude $\mathcal{M}$ is related to $h_{f i}{ }^{5}$

$$
\mathcal{M}=4(2 \pi)^{3} \sqrt{E_{A} E_{B} E_{C} E_{D}} h_{f i} .
$$

Using (22) in (21) in the laboratory system, one obtains

$$
\frac{d \sigma}{d \Omega}=\frac{16 \pi^{2} E_{K^{-}} E_{p} E_{\Lambda} E_{\eta}}{\left(E_{K^{-}}+E_{p}\right)\left(E_{\Lambda}+E_{\eta}\right)} \frac{\left|\vec{p}_{f}\right|}{\left|\vec{P}_{k}\right|}\left|h_{f i}\right|^{2},
$$

where $E_{k}$ are the energy particles and $m_{k^{-}}=0.493 \mathrm{GeV}, m_{p}=0.938 \mathrm{GeV}, m_{\eta}=$ $0.547 \mathrm{GeV}, m_{\Lambda}=1.11 \mathrm{GeV}$.

\section{Results}

Using Eq.(21) with $\sigma_{1}=3.5 \mathrm{GeV}, \sigma_{2}=0.2 \mathrm{GeV}, \alpha_{s}=0.7 \mathrm{GeV}, \alpha_{\lambda}=0.6 \mathrm{GeV}$, $x=0.7, \beta=0.7 \mathrm{GeV}$ and $b=0.2 \mathrm{GeV}^{2}$, we have found
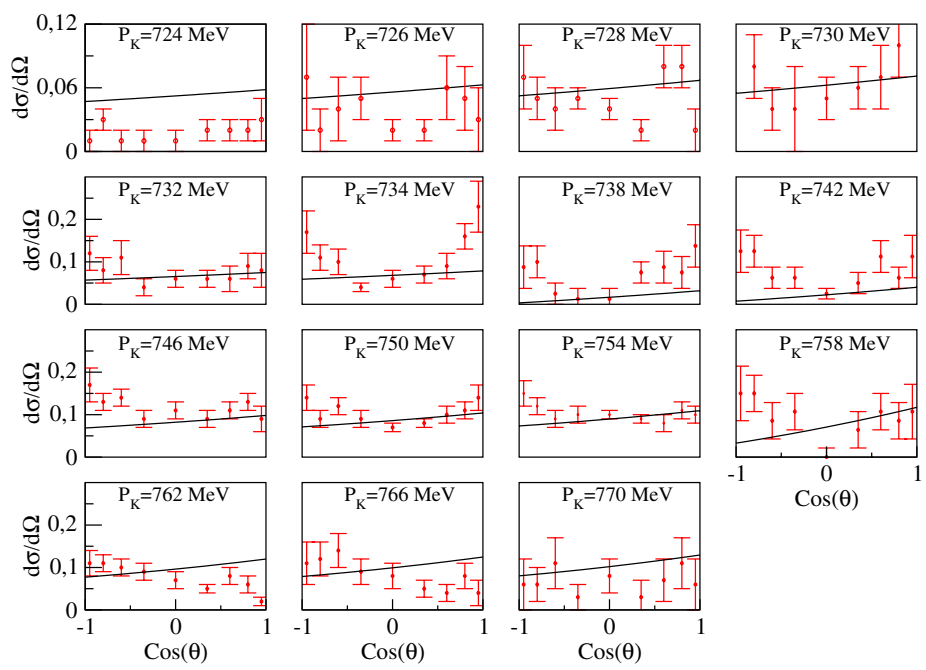

Fig. 2. Differential cross sections of the $K^{-}+p \rightarrow \Lambda+\eta$ compared with the data from Ref. ${ }^{6}$. The y-axis units are $m b / s r$. 


\section{B. C. Folador \& D. Hadjimichef}

The solid bold curves are for the model calculations and the dots are the data results. The overall fit is relatively close to experimental model even in the naive version of the model where the annihilation channels are not including. Our next step to improve our results is calculate the total cross-section and the differential cross sections of the $K^{-}+p \rightarrow \Lambda+\eta$ including annihilation. We also want to calculate $D N$ and $D^{*} N$ cross-sections.

\section{Acknowledgments}

This work was supported by Conselho Nacional de Desenvolvimento Científico e Tecnológico (CNPq) and Universidade Federal do Rio Grande do Sul (UFRGS).

\section{Appendix A. Wave-Functions}

The general meson wave-function is

$$
\Phi_{\alpha}^{\mu \nu}=\delta\left(\vec{p}_{\alpha}-\vec{p}_{\mu}-\vec{p}_{\nu}\right) \xi_{\alpha}^{f_{\mu} f_{\nu}} \Upsilon^{c_{\mu} c_{\nu}} \varphi\left(\vec{p}_{\mu}, \vec{p}_{\nu}\right)
$$

where $\xi_{\alpha}^{f_{\mu} f_{\nu}}$ is the spin-flavor part; $\Upsilon^{c_{\mu} c_{\nu}}$ is the color part given by

$$
\Upsilon^{c_{\mu} c_{\nu}}=\frac{\delta^{c_{\mu} c_{\nu}}}{\sqrt{3}}
$$

and $\varphi\left(\vec{p}_{\mu}, \vec{p}_{\nu}\right)$ is the spatial contribution, which is chosen

$$
\varphi\left(\vec{p}_{q}, \vec{p}_{\bar{q}}\right)=\left(\pi \beta^{2}\right)^{-\frac{3}{4}} \exp \left[-\frac{\left(m_{1} \vec{p}_{q}-m_{2} \vec{p}_{\bar{q}}\right)^{2}}{8 \beta^{2}}\right]
$$

with

$$
m_{1}=\frac{2 m_{\bar{q}}}{m_{q}+m_{\bar{q}}} \quad ; \quad m_{2}=\frac{2 m_{q}}{m_{q}+m_{\bar{q}}} .
$$

The baryon wave-function has a similar structure

$$
\Psi_{\alpha}^{\mu_{1} \mu_{2} \mu_{3}}=\delta\left(\vec{P}_{\alpha}-\sum_{i=1}^{3} \overrightarrow{p_{i}}\right) \xi_{\alpha}^{f_{1} f_{2} f_{3}} \Upsilon^{c_{1} c_{2} c_{3}} \Psi_{\overrightarrow{P_{\alpha}}}^{\overrightarrow{p_{2}} \overrightarrow{p_{3}}}
$$

where $\xi_{\alpha}^{f_{1} f_{2} f_{3}}$ is the spin-flavor part; $\Upsilon^{c_{1} c_{2} c_{3}}$ is the color part given by

$$
\Upsilon^{c_{1} c_{2} c_{3}}=\frac{\varepsilon^{c_{1} c_{2} c_{3}}}{\sqrt{6}} .
$$


Thus the wave-function is

$$
\begin{aligned}
\Psi_{\vec{P}}^{\overrightarrow{p_{1}}} \overrightarrow{p_{2}} \overrightarrow{p_{3}}= & \delta\left(\vec{P}-\vec{p}_{1}-\overrightarrow{p_{2}}-\overrightarrow{p_{3}}\right)\left(\frac{\sqrt{3}}{\pi \alpha_{\Lambda} \alpha_{\rho}}\right)^{3 / 2} \\
& \times \exp \left[-\frac{\alpha_{\Lambda}^{2}\left(\vec{p}_{1}-\vec{p}_{2}\right)^{2}+3 \alpha_{\rho}^{2}\left[M_{2} \vec{p}_{1}+M_{2} \vec{p}_{2}-2 M_{1} \vec{p}_{3}\right]^{2}}{4 \alpha_{\Lambda}^{2} \alpha_{\rho}^{2}}\right],
\end{aligned}
$$

or equivalently defining $x=\alpha_{\rho} / \alpha_{\Lambda}$

$$
\begin{aligned}
\Psi_{\vec{P}}^{\overrightarrow{p_{1}} \overrightarrow{p_{3}}}= & \delta\left(\vec{P}-\vec{p}_{1}-\vec{p}_{2}-\overrightarrow{p_{3}}\right)\left(\frac{\sqrt{3}}{\pi x \alpha_{\Lambda}^{2}}\right)^{3 / 2} \\
& \times \exp \left[-\frac{\left(\vec{p}_{1}-\vec{p}_{2}\right)^{2}+3 x^{2}\left[M_{2} \vec{p}_{1}+M_{2} \vec{p}_{2}-2 M_{1} \vec{p}_{3}\right]^{2}}{4 \alpha_{\Lambda}^{2} x^{2}}\right] .
\end{aligned}
$$

A simplification that can be introduced is to consider $x=1$,

$$
\alpha_{\lambda}=\alpha_{\rho} \equiv \alpha \text {. }
$$

On that way the wave-function (A.8) becomes

$$
\begin{aligned}
\Psi_{\vec{P}}^{\overrightarrow{p_{1}}} \overrightarrow{p_{3}}= & \delta\left(\vec{P}-\vec{p}_{1}-\overrightarrow{p_{2}}-\overrightarrow{p_{3}}\right)\left(\frac{\sqrt{3}}{\pi \alpha^{2}}\right)^{3 / 2} \\
& \times \exp \left[-\frac{\left(\vec{p}_{1}-\vec{p}_{2}\right)^{2}+3\left[M_{2} \vec{p}_{1}+M_{2} \vec{p}_{2}-2 M_{1} \vec{p}_{3}\right]^{2}}{4 \alpha^{2}}\right] .
\end{aligned}
$$

On the nucleons particular case,

$$
m_{l}=m_{p} \quad \rightarrow \quad M_{1}=M_{2}=\frac{1}{3}
$$

the wave-function (A.10) reduces to the well-known result of the Quark Model

$$
\begin{aligned}
\Psi_{\vec{P}}^{\overrightarrow{p_{1}}} \overrightarrow{p_{2}} \overrightarrow{p_{3}}= & \delta\left(\vec{P}-\vec{p}_{1}-\vec{p}_{2}-\vec{p}_{3}\right)\left(\frac{\sqrt{3}}{\pi \alpha^{2}}\right)^{3 / 2} \\
& \times \exp \left[\frac{-p_{1}^{2}-p_{2}^{2}-p_{3}^{2}+\vec{p}_{1} \cdot \vec{p}_{2}+\vec{p}_{1} \cdot \vec{p}_{3}+\vec{p}_{2} \cdot \vec{p}_{3}}{3 \alpha^{2}}\right] .
\end{aligned}
$$

\section{References}

1. D. Hadjimichef, G. Krein, S. Szpigel, and J. S. da Veiga, Ann. of Phys. 268 n.1, 105 (1998).

2. D. Hadjimichef, J. Haidenbauer, and G. Krein, Phys.Rev. C63, 035204 (2001); e-Print Archive: nucl-th/0010044(2000).

3. D. Hadjimichef, J. Haidenbauer, and G. Krein, Phys.Rev. C66, 055214 (2002).

4. K. A. Olive et al. (Particle Data Group), Chin. Phys. C 38, 090001 (2014).

5. T. Barnes and E. S. Swanson, Phys. Rev. D 46, 131 (1992).

6. A. Starostin et al, Phys. Rev. C64, 055205 (2001).

7. L. Y. Xiao and X. H. Zhong, Phys. Rev. C88, 065201 (2013). 